\title{
La gestione dell'iperfosfatemia
}

\author{
Maurizio Gallieni ${ }^{1,2}$ \\ ${ }^{1}$ U.O. Nefrologia e Dialisi, Presidio Ospedaliero San Carlo Borromeo, ASST Santi Paolo e Carlo, Milano \\ ${ }^{2}$ Dipartimento di Scienze Biomediche e Cliniche "L. Sacco", Università degli Studi di Milano, Milano
}

\begin{abstract}
Management of hyperphosphatemia
Serum phosphate levels, along with serum calcium and parathyroid hormone (PTH), are the most commonly used markers for the diagnosis, monitoring and treatment of chronic kidney disease (CKD)-mineral and bone disorder (MBD) abnormalities in patients affected by kidney failure. Hyperphosphatemia appears late in the course of CKD, but the consequences of phosphate retention, such as increased levels of FGF23 and hyperparathyroidism, can be appreciated much earlier. Clinical practice guidelines suggest aiming at phosphate levels that currently are reached by no more than $50 \%$ of patients, despite major efforts of nephrologists, nurses and dieticians in addressing the issue of dietary and pharmacological compliance. Thus, there are margins of improvement of phosphate management in CKD patients, which could be accomplished by dietary and/or pharmacological approaches aimed at improving patient compliance. Severe hyperphosphatemia is always associated with phosphate overload, but it should be kept in mind that even mild hyperphosphatemia can be linked to a positive phosphate balance and its deleterious consequences. In fact, hyperphosphatemia is associated with mortality and morbidity (including hyperparathyroidism, left ventricular hypertrophy, and vascular calcifications). Management of hyperphosphatemia should take into account the main rings of the therapeutic chain of phosphate control: defense of residual renal function, adequate dialysis, diet, and phosphate binders. Along with the traditional approaches to phosphate control, in this article new aspects of phosphate control are summarized, such as cooking tips, education regarding the presence of phosphate-based additives/preservatives in foods and even drugs, and the potential advantages of last-generation phosphate binders.
\end{abstract}

Keywords: Additives, CKD, Dialysis, Diet, Phosphate, Phosphate binders, PTH

L'omeostasi del fosforo è marcatamente alterata nei pazienti con malattia renale cronica (CKD) già nelle prime fasi della malattia, per la riduzione della capacità di escrezione renale del fosforo. Tuttavia, tranne che negli stadi più avanzati di CKD, l'organismo mette in atto diversi meccanismi compensatori che consentono di mantenere nei limiti di norma i livelli plasmatici di fosforo. Questo fenomeno era già stato descritto nel 1973 da Slatopolsky e Bricker (1) con la formulazione della trade-off hypothesis secondo la quale il mantenimento di una fosfatemia normale veniva pagato con un aumento dei livelli di paratormone (PTH), grazie alla sua azione fosfaturica. Negli anni successivi sono stati identificati ulteriori meccanismi e

Accepted: August 23, 2016

Published online: September 5, 2016

Indirizzo per la corrispondenza:

Dr. Maurizio Gallieni

U.O. Nefrologia e Dialisi

Presidio Ospedaliero San Carlo Borromeo

Via Pio II, 3

20153 Milano

email: maurizio.gallieni@unimi.it fattori importanti di modulazione dell'omeostasi del fosforo, tra cui il sistema vitamina D e più recentemente FGF23 (Fibroblast Growth Factor 23) (2).

Pur considerando la complessità del sistema di modulazione del metabolismo calcio-fosforo nel soggetto normale e ancor più nel paziente con CKD, uno studio semplice ma fondamentale di Slatopolsky et al (3) ha dimostrato molti anni fa che riducendo significativamente l'introito dietetico di fosforo si può prevenire molto efficacemente l'iperparatiroidismo (1). Negli ultimi anni, peraltro, l'attenzione al controllo dell'iperfosfatemia si è accentuata in quanto oltre all'iperparatiroidismo sono emersi altri esiti clinici rilevanti associati al fosforo. In particolare, nei pazienti in dialisi l'iperfosfatemia si associa ad aumento dell'ipertrofia ventricolare sinistra (4) e della mortalità cardiovascolare e per tutte le cause $(5,6)$. Persino nei soggetti con funzione renale normale livelli di fosforo più elevati si associano a esiti clinici più sfavorevoli (7-9).

L'iperfosfatemia compare negli stadi più avanzati di CKD, in genere quando il filtrato glomerulare scende sotto i $30 \mathrm{~mL} /$ min. Il problema della gestione dell'iperfosfatemia diventa molto rilevante nello stadio 5 , sotto i $15 \mathrm{~mL} / \mathrm{min}$ e in particolare nei pazienti sottoposti a dialisi. Tuttavia, una riduzione dell'introito di fosforo proporzionale alla riduzione del filtrato 
glomerulare, prima che si sviluppi iperfosfatemia, finalizzata al mantenimento di un bilancio corporeo neutro di fosforo è probabilmente l'approccio più efficace nel prevenire le complicanze tipiche della CKD-MBD (Chronic Kidney Disease Mineral and Bone Disorder) e verosimilmente la morbilità e mortalità cardiovascolare. Tuttavia, l'effetto dell'iperfosfatemia sulla mortalità richiede probabilmente tempi medio-lunghi. Infatti, uno studio su 10672 pazienti con CKD (filtrato glomerulare $<60 \mathrm{~mL} / \mathrm{min}$ ) non in dialisi con una durata mediana dell'osservazione di 27 mesi non ha evidenziato un'associazione indipendente della fosfatemia con il rischio di morte o di progressione verso la dialisi (10). D'altra parte, analizzando in una metanalisi anche studi con un follow-up più lungo, Da et al hanno concluso che esiste un'associazione indipendente tra fosfatemia e mortalità, come pure una maggiore propensione alla necessità di trattamento dialitico, tra pazienti con CKD. In particolare, per ogni aumento di fosfatemia di $1 \mathrm{mg} / \mathrm{dL}$ il rischio di dialisi aumenta del $36 \%$ e il rischio di morte del $20 \%$ (11).

In sostanza, è importante ricordare che basarsi sulla fosfatemia per valutare l'efficacia dell'approccio terapeutico può essere fuorviante. I pazienti iperfosfatemici richiedono molta attenzione perché verosimilmente hanno un apporto alimentare superiore al desiderato, ma vanno considerate cause alternative, come un iperparatiroidismo mal controllato, un eccesso di trattamento con vitamina $D$ e soprattutto un'inadeguata rimozione di fosforo con la dialisi. D'altra parte è possibile che si sviluppi un bilancio positivo di fosforo anche con fosforemie lievemente aumentate, il che può associarsi a esiti sfavorevoli (Fig. 1).

Peraltro, anche solo attenendosi ai livelli di fosfatemia indicati dalle linee guida, i risultati ottenuti nella pratica clinica non sono soddisfacenti, evidenziando un fenomeno di inerzia terapeutica (12). Una valutazione delle modalità di trattamento delle alterazioni del metabolismo minerale in 727 pazienti affetti da CKD in stadio 3b-5 non in dialisi, seguiti in 19 Centri nefrologici italiani, ha evidenziato che la maggior parte dei pazienti (85\%) aveva livelli di fosfatemia compresi nel target terapeutico. Solo una minoranza di pazienti $(17,3 \%)$ veniva trattato con chelanti del fosforo $(15,5 \%$ sali di calcio, $1,8 \%$ sali di alluminio). Tra i pazienti iperfosfatemici visitati al tempo zero e dopo 6 mesi, l'inerzia terapeutica (ovvero nessun provvedimento terapeutico nonostante il valore di fosforo non rientrasse nei limiti desiderati) era del 54\%, più frequentemente nei pazienti in stadio 3b CKD. Inoltre l'iperparatiroidismo, un segno indiretto di verosimile bilancio positivo di fosforo, era presente nel $65 \%$ dei pazienti di questa popolazione.

Isakova et al (13) hanno ipotizzato di effettuare studi clinici randomizzati nei pazienti con CKD non in dialisi sul controllo del fosforo e sugli esiti clinici conseguenti. Per ovviare al fatto che la maggior parte di questi pazienti ha fosfatemie nei limiti di norma, propongono di utilizzare un test surrogato di sovraccarico di fosforo, come è l'emoglobina glicata rispetto alla glicemia per il diabete. Tale fattore surrogato potrebbe essere il livello di PTH o di FGF23 (13).

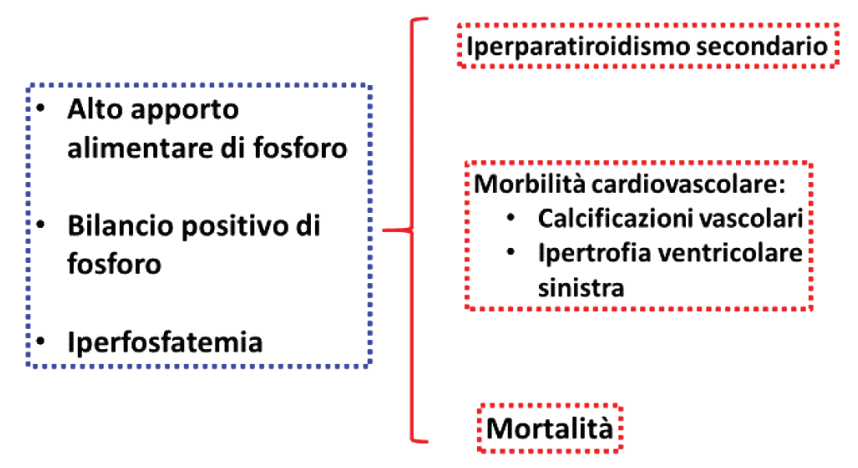

Fig. 1 - Aspetti diversi del sovraccarico di fosforo nei pazienti con malattia renale cronica (a sinistra) e principali esiti clinici (a destra). L'iperfosfatemia è il parametro più semplice da identificare, ma spesso anche pazienti con fosfatemie più contenute, anche non dializzati, hanno bilanci di fosforo positivi per un contenuto elevato di fosfati nella dieta.

\section{Il bilancio del fosforo}

Nel soggetto con normale funzione renale il bilancio tra assorbimento intestinale ed escrezione renale di fosforo è neutro ed è regolato finemente comprendendo anche l'equilibrio tra formazione e riassorbimento osseo, un'altra importante fonte di fosfati endogeni. II sistema scheletrico rappresenta la riserva principale di fosfati e contiene oltre l' $80 \%$ del fosforo corporeo. Da un punto di vista funzionale, è più utile però considerare il pool di fosforo "scambiabile", che è distribuito nell'organismo in tre compartimenti: il 70\% all'interno delle cellule, dove svolge un ruolo cruciale nelle reazioni di fosforilazione indispensabili per il corretto funzionamento delle cellule e dell'organismo; il $29 \%$ sul fronte di mineralizzazione dell'osso e meno dell' $1 \%$ si trova nel siero dove può essere misurato con un prelievo ematico. Nell'insufficienza renale, quando i meccanismi compensatori si esauriscono e si sviluppa un bilancio di fosfati positivo per un deficit di escrezione renale, il livello ematico di fosforo aumenta (14). Si osserva inoltre un deposito di calcio e fosforo nei tessuti molli e in particolare nelle pareti dei vasi arteriosi (15), associato a un depauperamento di mineralizzazione dell'osso. Come è noto a tutti i clinici, anche se non tutti concordano sulla loro pericolosità, la prevalenza di calcificazioni vascolari è molto elevata nella CKD, ovvero del $40 \%$ nei pazienti in stadio 5 non in dialisi (16) e dell' $83 \%$ nei pazienti dializzati (17).

La gestione dell'iperfosfatemia nel paziente con CKD deve basarsi sui tre aspetti che consentono di influire sul bilancio corporeo di fosforo, vale a dire la nutrizione, l'uso di prodotti che blocchino (chelanti) l'assorbimento intestinale del fosforo e, nel caso dei pazienti con malattia renale avanzata, la rimozione di fosforo con la dialisi. Molto spesso i risultati sono inadeguati e si sente il bisogno di miglioramenti terapeutici che consentano di raggiungere l'equilibrio tra introito ed eliminazione dei fosfati.

Analizziamo quindi alcuni degli aspetti più rilevanti della gestione dell'iperfosfatemia. 


\section{La nutrizione}

La quantità di fosforo alimentare consigliata per i pazienti affetti da CKD è di soli $700 \mathrm{mg}$ al giorno, ma l'effettivo introito varia tra 1000 e 2000 mg/die (18). Il fosforo introdotto con la dieta è strettamente correlato alla quantità di proteine. È stato calcolato che una dieta contenente $60 \mathrm{~g} /$ die di proteine fornisce circa $800 \mathrm{mg}$ di fosforo e una dieta contenente $90 \mathrm{~g}$ di proteine contiene circa $1200 \mathrm{mg}$ di fosforo (19).

Nel soggetto normale, la fosfaturia corrisponde a un assorbimento intestinale netto di fosforo del $65 \%$, che è influenzato dal calcitriolo, il metabolita attivo del sistema vitamina D. I principali regolatori della fosfaturia sono PTH e FGF23. Nel paziente con CKD si osserva una riduzione della capacità di escrezione renale del fosforo e anche una riduzione del suo assorbimento intestinale, per il concomitante deficit di sintesi del calcitriolo. Tuttavia, il bilancio di fosforo tende a diventare sempre più positivo con il progredire della malattia renale cronica e la terapia con vitamina $\mathrm{D}$, utilizzata per normalizzare i livelli di calcio e di PTH, può accentuare la tendenza alla ritenzione di fosforo. Lo studio più noto sull'assorbimento intestinale di fosforo nei pazienti emodializzati indica una percentuale di assorbimento media del $60 \%$. Tuttavia, nei 5 pazienti studiati la variabilità di assorbimento era notevole (26\%, 40\%, 69\%, 84\% e $85 \%$ ) (20). $\mathrm{Va}$ anche ricordato che una dieta a basso contenuto di fosforo può purtroppo stimolare il suo assorbimento intestinale (21), vanificando in parte gli sforzi dietetici.

Pertanto, la nutrizione rappresenta un ambito di grande importanza, non ancora del tutto esplorato, per poter adattare il carico intestinale di fosforo all'alterato sistema di regolazione della fosfatemia, senza indurre deficit di calorie o di nutrienti. È infatti molto importante essere cauti nella restrizione dietetica dei cibi contenenti fosforo per evitare di indurre malnutrizione nei pazienti con CKD. Tuttavia, negli ultimi anni sono emerse importanti differenze qualitative tra gli alimenti contenenti fosforo e la scelta del tipo di alimenti può avere un'importanza anche maggiore della riduzione quantitativa del fosforo nella dieta, in quanto alcuni cibi lavorati hanno una quantità di fosforo molto più elevata rispetto agli stessi cibi freschi, per effetto dell'uso di additivi a base di fosforo (22). Tali additivi sono particolarmente comuni nei cibi congelati ( $72 \%$ dei cibi contengono additivi), nelle carni confezionate (65\%), nei prodotti da forno (57\%), zuppe pronte (54\%) e yogurt (51\%) (23). Una dieta che comprende principalmente cibi contenenti additivi a base di fosforo, rispetto a una dieta priva di additivi, fornisce in media una quota aggiuntiva di fosforo corrispondente a $736 \mathrm{mg} / \mathrm{die}$ (23). Paradossalmente, pur essendo cibi lavorati questi alimenti costano meno degli alimenti freschi e pertanto i consumatori sono invogliati al loro acquisto anche per motivi economici.

Un aspetto particolare e poco conosciuto del carico di fosforo da additivi è quello relativo al fosforo contenuto nei medicinali, definito il "carico di fosforo iatrogeno" (24). Sebbene nella scheda tecnica del prodotto sia indicato quando è presente il fosforo, in genere le quantità non sono indicate. Tra i 200 farmaci più prescritti ai pazienti in dialisi, 23 (11,5\%) contengono fosforo aggiunto tra gli eccipienti, in alcuni casi in quantità molto significative, come riscontrato per la paroxetina $40 \mathrm{mg}$ (111 mg di P/cpr), per una formulazione generica di lisinopril $10 \mathrm{mg}$ (33 mg di P/cpr) e di amlodipina $10 \mathrm{mg}$ (40 mg di P/cpr) (24).

Un altro importante aspetto qualitativo che influenza la quota di fosforo assorbito dall'intestino è l'origine vegetale o animale. Infatti, la quota assorbita è minima per il fosforo contenuto nei vegetali sotto forma di fitato, intermedia per gli alimenti di origine animale ed è massima per i fosfati o i polifosfati contenuti negli additivi alimentari (25). La variabilità dell'assorbimento intestinale di fosforo in base all'origine animale o vegetale delle proteine è stata studiata molto bene da Moe et al (26). In pazienti con CKD (GFR medio $32 \mathrm{~mL} / \mathrm{min}$ ) sono stati confrontati con un disegno a crossover una dieta vegetariana e una dieta contenente carni, con lo stesso quantitativo di fosforo, assunte per una settimana. Nelle ultime 24 ore di ognuno dei due periodi di 7 giorni i pazienti venivano ricoverati in un reparto di ricerca clinica per un controllo frequente degli esami ematici e urinari. La dieta vegetariana induceva una riduzione significativa dei livelli di fosforo e di FGF23 e anche le variazioni circadiane del fosforo erano più favorevoli alla fine del periodo con dieta vegetariana. Questo studio dimostra quindi che la fonte delle proteine ha un effetto significativo sull'assorbimento e sull'omeostasi del fosforo nei pazienti con CKD.

Oltre al vantaggio dell'utilizzo di proteine di origine vegetale, è utile scegliere cibi con un basso rapporto fosforo/proteine. Un tipico esempio ben noto ai nefrologi, ma che deve sempre essere comunicato ai pazienti, è rappresentato dall'uovo. Il tuorlo contiene la maggior parte del fosforo (in forma di fosfolipidi e quindi con un basso contenuto di proteine), mentre l'albume contiene una buona quantità di proteine $(3,7$ g per ogni uovo) e una quantità minima di fosforo. II bianco dell'uovo è quindi una fonte di proteine di alto valore biologico, sostanzialmente priva di fosforo. Pertanto, l'utilizzo di ricette che comprendano l'uso o l'aggiunta del bianco dell'uovo consente di superare favorevolmente la stretta relazione esistente tra quantità di proteine e quantità di fosforo, aumentando la quota proteica senza aumentare significativamente la quantità di fosforo $(27,28)$.

Un ulteriore metodo per ridurre l'assorbimento intestinale di fosforo è la cottura degli alimenti. Bollire gli alimenti o cuocerli (stufare) in olio e acqua riduce sostanzialmente il contenuto di fosforo negli alimenti senza modificarne il contenuto in proteine. Ad esempio, stufare in olio con una piccola quantità di acqua dei filetti di petto di pollo riduce del $24 \%$ la quantità di fosforo, consentendo di migliorare i menù per i pazienti dializzati (29).

Infine, sono emersi anche suggerimenti sul possibile ruolo del microbiota intestinale e della supplementazione con fermenti lattici nel controllo dell'assorbimento intestinale di fosfati (30).

In sintesi, va quindi ricordato che il fosforo contenuto negli additivi e negli alimenti di origine animale viene assorbito in misura superiore rispetto al fosforo di origine vegetale. In particolare dal $40 \%$ al $60 \%$ del fosforo contenuto negli alimen- 
ti finisce nel circolo sanguigno, mentre il fosforo aggiunto agli alimenti come additivo viene assorbito dal $90 \%$ al $100 \%$ (25). I consigli dietetici ai pazienti con CKD devono includere informazioni non solo sulla quantità di fosfati ma anche sulla fonte (animale, vegetale, additivi) da cui essi provengono. Vanno poi considerati i cibi con il miglior rapporto fosforo/proteine (28) e le modalità di cottura degli alimenti (29). L'approccio dietetico ottimale comprende frequenti colloqui sull'argomento fosforo (con il nefrologo e/o il dietologo e/o il dietista), un'adeguata informazione sui cibi ricchi di fosforo e sulla loro fonte animale o vegetale e un'attenzione particolare all'importanza di evitare cibi e bevande che contengano additivi a base di fosfati (31).

\section{Dialisi}

Il secondo aspetto fondamentale nella gestione del bilancio del fosforo consiste nella sua rimozione dialitica. Tutte le metodiche dialitiche rimuovono quantità significative di fosforo, ma solo l'emodialisi protratta per molte ore (notturna e/o quotidiana) consente di rimuovere tutto il fosforo ingerito con la dieta evitando l'uso di chelanti del fosforo (32). La rimozione di fosforo dipende quindi principalmente dal tempo di trattamento, ma dato che per i fosfati la depurazione è più elevata nelle prime due ore di dialisi anche una dialisi quotidiana, a parità di tempo di trattamento settimanale, è più efficiente rispetto alla dialisi trisettimanale (33).

La distribuzione corporea multi-compartimentale del fosforo e il suo lento passaggio dal compartimento intracellulare all'extracellulare e al plasma durante la seduta dialitica, riduce l'efficienza della rimozione di fosforo e determina un significativo rebound post-dialisi $(34,35)$.

Ipotizzando un introito alimentare contenuto a $7 \mathrm{~g} / \mathrm{settimana}$ (1000 mg/die), assorbiti per il 50-60\%, la quota di fosfati da eliminare sarebbe di 3,5-4,2 g/settimana. La Tabella I indica il range della quantità di fosfati rimossa ogni settimana con diverse strategie dialitiche (28). L'emodiafiltrazione in post-diluizione consente una rimozione di fosforo superiore di circa il $20 \%$ rispetto all'emodialisi convenzionale $(35,36)$, sebbene questa differenza si attenui per valori di fosforemia più elevati. L'importanza del livello plasmatico di fosforo nella valutazione del ruolo della dialisi nel mantenimento di un corretto bilancio di fosforo non è sempre tenuta in adeguata considerazione. Nello studio di Lornoy et al (36), la rimozione media di fosforo era di $951 \mathrm{mg} /$ dialisi nei pazienti con fosfatemie comprese tra 4,2 e $6,4 \mathrm{mg} / \mathrm{dL}$, rispetto a $607 \mathrm{mg} /$ dialisi nei pazienti con fosfatemie più basse e a 1435 $\mathrm{mg} /$ dialisi in quelli con fosfatemie più elevate.

In sintesi, in emodialisi l'utilizzo di metodiche miste diffusivo-convettive e l'aumento del numero e/o della durata delle sessioni dialitiche sono tutte misure in grado di migliorare la rimozione dialitica di fosfati.

La dialisi peritoneale può consentire una buona rimozione di fosforo, soprattutto nei pazienti con una buona efficienza di trasporto della membrana peritoneale e nei pazienti con funzione renale residua. Nei pazienti anurici, i bassi trasportatori
TABELLA I - Quantità di fosfati (grammi/settimana, range) rimossa con diverse strategie dialitiche

Fosfati assorbiti con introito alimentare di $7 \mathrm{~g} /$ $3,5-4,2 \mathrm{~g}$

settimana (1000 mg/die), senza chelanti

Emodialisi convenzionale diffusiva, 4 ore

$2,3-2,6 \mathrm{~g}$

Emodialisi diffusiva estesa, $\geq 5$ ore

$3,0-3,6 \mathrm{~g}$

Emodialisi notturna, 8 ore

$4,5-4,9 \mathrm{~g}$

Emofiltrazione endogena con reinfusione, 4 ore

$1,8-2,4 \mathrm{~g}$

Emodiafiltrazione in post-diluizione, 4 ore

$3,0-3,3 \mathrm{~g}$

Emofiltrazione in pre-diluizione

$0,9-1,5 \mathrm{~g}$

(volume di scambio: peso corporeo $\times 1,2$ )

Dialisi peritoneale (CAPD, $2 \mathrm{~L} \times 4 /$ die) $2,0-2,2 \mathrm{~g}$

Modificata da Cupisti A, Gallieni M, Rizzo MA, Caria S, Meola M, Bolasco P. Phosphate control in dialysis. Int J Nephrol Renovasc Dis. 2013; 6:193-205 (28).

rimuovono meglio il fosforo in CAPD (Continuous Ambulatory Peritoneal Dialysis). Nei pazienti trattati con APD (Automated Peritoneal Dialysis) che sviluppano iperfosfatemia e presentano una rimozione peritoneale insufficiente, è possibile migliorare la rimozione dialitica aumentando i tempi di sosta o spostando il paziente alla metodica manuale (28).

\section{Chelanti del fosforo}

La Tabella II indica le caratteristiche del chelante del fosforo ideale. I chelanti del fosforo influiscono positivamente nell'evoluzione delle CKD in quanto consentono una riduzione dell'assorbimento intestinale di fosforo e il loro uso è stato associato a una riduzione della morbilità e mortalità (37-39). Lo studio osservazionale europeo Cosmos, che ha coinvolto 6797 pazienti seguiti in modo prospettico per 3 anni, ha dimostrato che nei pazienti a cui vengono prescritti chelanti del fosforo si osserva una riduzione della mortalità globale del $29 \%$ e una riduzione della mortalità cardiovascolare del 22\% (39).

In contrasto, una recente metanalisi (40) conclude che non ci siano prove che i chelanti del fosforo, rispetto al placebo, riducano la mortalità nei pazienti con CKD, sebbene sia stato evidenziato un vantaggio di sopravvivenza analizzando complessivamente gli studi di confronto tra sevelamer e sali di calcio. Questa osservazione conferma i risultati di una pre-

TABELLA II - Chelante del fosforo ideale

- Elevata efficacia nel legare il fosforo

- Ben tollerato, con pochi effetti collaterali e senza potenziali tossicità

- Associato a buona compliance del paziente

- Assenza di interazioni (o minime interazioni) con altri farmaci

- $\quad$ Basso costo 
cedente revisione sistematica che confrontava i chelanti privi di calcio rispetto a quelli a base di calcio (41). Una lettura attenta del lavoro di Palmer et al (40) evidenzia tuttavia che il numero di studi di confronto tra chelanti del fosforo e placebo era alquanto limitato (4 studi in cui fosse stata pre-specificata un'analisi di sopravvivenza, il maggiore dei quali aveva una popolazione con età media di 49 anni) e la durata di questi studi era al massimo di 3 mesi. L'affermazione che non ci sia vantaggio nell'uso dei chelanti rispetto al placebo per quanto riguarda la mortalità è quindi da prendere con molta prudenza.

Per quanto riguarda i sali di calcio, sempre secondo i risultati di questa metanalisi, non è chiaro se possano essere dannosi rispetto ai chelanti privi di calcio e/o al placebo (40). I chelanti a base di calcio possono indurre ipercalcemia e/o determinare un bilancio corporeo di calcio positivo e quindi un sovraccarico intracellulare, oltre a facilitare il processo di calcificazione dei tessuti molli, in particolare le pareti arteriose. Nei pazienti con CKD la presenza di ipercalcemia e di sovraccarico di calcio possono aumentare il rischio di aritmie sopra-ventricolari e ventricolari, fino all'arresto cardiaco (42).

Pertanto non è consigliabile utilizzare dosi elevate di sali di calcio, mentre dosi modeste (fino a $1 \mathrm{~g} /$ die di calcio-elemento) possono rappresentare un approccio iniziale adeguato per ridurre l'assorbimento intestinale di fosforo e un chelante privo di calcio può essere associato in un secondo momento, se la risposta clinica non è soddisfacente e/o nei pazienti che sono gravati da rilevanti calcificazioni vascolari (43). In effetti i pazienti con calcificazioni vascolari gravi hanno una sopravvivenza del $20 \%$ a 6 anni, rispetto al 95\% dei pazienti senza calcificazioni (44).

L'uso dei chelanti del fosforo pone anche il problema del numero di compresse e quindi dell'aderenza al trattamento. Non è infrequente che un paziente dializzato debba assumere 15-20 compresse al giorno (nel $25 \%$ dei pazienti oltre 25 compresse al giorno), rappresentate in buona parte dai chelanti del fosforo (45). L'aderenza alle terapie dirette al controllo della fosfatemia può essere valutata con metodi diversi ma risulta comunque non adeguata, sia che si considerino i livelli plasmatici di fosforo (aderenza $58 \%$ ) che le misure di autovalutazione dell'aderenza (31\%) (46).

Per migliorare i risultati clinici e l'aderenza alla terapia è quindi molto importante ridurre il numero di compresse da assumere e utilizzare un chelante molto efficiente. Palmer et al (40) hanno indicato che i chelanti a base di ferro hanno dimostrato una maggior capacità di riduzione della fosforemia e che pertanto meritino un approfondimento nell'ambito di studi clinici controllati.

Riprendendo l'esempio di un paziente che assume una dieta contenente $1000 \mathrm{mg} /$ die di fosforo e quindi abbia un assorbimento di $600 \mathrm{mg} / \mathrm{die}$, con una rimozione dialitica settimanale di 2,8 grammi (rimozione media giornaliera $400 \mathrm{mg}$ ), sarà necessario bloccare l'assorbimento intestinale di $200 \mathrm{mg} /$ die di fosforo. La quantità di fosforo bloccata nell'intestino da chelanti utilizzati a dosi piene è di circa $170 \mathrm{mg} /$ die $(47,48)$. In particolare le dosi medie riportate sono di 5,9 g/die di calcio acetato, 6,3 g/die di sevelamer carbonato, e 2,7 g/die di lantanio carbonato (48). Di conse- guenza, l'introduzione del chelante sucroferrico, con la sua maggiore efficienza e quindi con un numero ridotto di compresse da assumere, rappresenta un vantaggio clinico (40).

\section{Conclusioni}

Lo studio osservazionale Cosmos (49) ha indicato che il $52 \%$ dei pazienti dializzati ha fosforemie nel range suggerito dalle linee guida Kidney Disease Outcomes Quality Initiative (KDOQI) e solo il $27 \%$ dei pazienti rientra nel range suggerito dalle linee guida Kidney Disease: Improving Global Outcomes (KDIGO) ci sono quindi ampi margini di miglioramento nella gestione dei pazienti con iperfosfatemia. L'iperfosfatemia o più precisamente il sovraccarico di fosforo anche con fosfatemie apparentemente ben controllate (risultato della combinazione di intake dietetico, blocco del suo assorbimento intestinale, funzione renale residua e rimozione dialitica) si associa a morbilità (compresa l'ipertrofia ventricolare sinistra) e mortalità nei pazienti con CKD e secondo alcuni studi anche nei soggetti sani. Nei pazienti con CKD si associa inoltre a iperparatiroidismo e calcificazioni vascolari.

La pratica medica attuale considera la fosforemia il solo marcatore per diagnosticare, monitorare e trattare le anomalie del bilancio del fosforo nei pazienti con malattia renale cronica. Tuttavia, le conseguenze di squilibri del bilancio del fosforo sono evidenti nei pazienti con CKD, in quanto numerose alterazioni del metabolismo minerale si osservano prima dell'innalzamento della fosforemia.

Le implicazioni cliniche potenziali sono notevoli (50). Agli approcci tradizionali di gestione dell'iperfosfatemia (riduzione del contenuto dietetico, uso di chelanti, efficienza della dialisi) vanno associati accorgimenti emersi più di recente, come le modalità di cottura degli alimenti, l'attenzione alla presenza negli alimenti e anche nei farmaci di additivi/conservanti a base di fosforo, i potenziali vantaggi dei chelanti di ultima generazione, l'attenzione al problema dell'aderenza ai trattamenti.

\section{Disclosures}

Financial support: This Supplement has been supported by an unrestricted educational grant provided by Vifor Fresenius Medical Care Renal Pharma Italia S.r.l.

Conflict of interest: The author has no conflict of interest.

\section{Bibliografia}

1. Slatopolsky E, Bricker NS. The role of phosphorus restriction in the prevention of secondary hyperparathyroidism in chronic renal disease. Kidney Int. 1973;4(2):141-5.

2. Bergwitz $\mathrm{C}$, Jüppner $\mathrm{H}$. Regulation of phosphate homeostasis by PTH, vitamin D, and FGF23. Annu Rev Med. 2010;61:91-104.

3. Slatopolsky E, Caglar S, Pennell JP, et al. On the pathogenesis of hyperparathyroidism in chronic experimental renal insufficiency in the dog. J Clin Invest. 1971;50(3):492-9.

4. Gallieni M, Pedone M. Left ventricular hypertrophy and serum phosphate in peritoneal dialysis patients. Kidney Int. 2013;84(4):850. 
5. Kestenbaum B. Serum phosphate levels and mortality risk among people with chronic kidney disease. J Am Soc Nephrol. 2005; 16:520-8.

6. Tentori F, Blayney MJ, Albert JM, et al. Mortality risk for dialysis patients with different levels of serum calcium, phosphorus, and PTH: the Dialysis Outcomes and Practice Patterns Study (DOPPS). Am J Kidney Dis. 2008;52(3):519-30.

7. Dhingra R, Sullivan LM, Fox CS, et al. Relations of serum phosphorus and calcium levels to the incidence of cardiovascular disease in the community. Arch Intern Med. 2007;167:879-85.

8. Tonelli M, Sacks F, Pfeffer M, et al. Relation between serum phosphate level and cardiovascular event rate in people with coronary disease. Circulation. 2005;112:2627-33.

9. Foley RN. Phosphate levels and cardiovascular disease in the general population. Clin J Am Soc Nephrol. 2009;4:1136-9.

10. Mehrotra R, Peralta CA, Chen SC, et al. No independent association of serum phosphorus with risk for death or progression to end-stage renal disease in a large screen for chronic kidney disease. Kidney Int. 2013;84(5):898-7.

11. Da J, Xie X, Wolf $M$, et al. Serum phosphorus and progression of CKD and mortality: a meta-analysis of cohort studies. Am J Kidney Dis. 2015;66(2):258-65.

12. Gallieni M, De Nicola L, Santoro D, et al. Management of CKDMBD in non-dialysis patients under regular nephrology care: a prospective multicenter study. J Nephrol. 2016;29(1):71-8.

13. Isakova T, Gutiérrez OM, Wolf M. A blueprint for randomized trials targeting phosphorus metabolism in chronic kidney disease. Kidney Int. 2009;76(7):705-16.

14. Hruska KA, Mathew S, Lund R, Qiu P, Pratt R. Hyperphosphatemia of chronic kidney disease. Kidney Int. 2008;74(2):148-57.

15. Giachelli CM. The emerging role of phosphate in vascular calcification. Kidney Int. 2009;75(9):890-7.

16. Russo D, Palmiero G, De Blasio AP, Balletta MM, Andreucci VE. Coronary artery calcification in patients with CRF not undergoing dialysis. Am J Kidney Dis. 2004;44(6):1024-30.

17. Chertow GM, Burke SK, Raggi P; Treat to Goal Working Group. Sevelamer attenuates the progression of coronary and aortic calcification in hemodialysis patients. Kidney Int. 2002;62(1):245-52.

18. Kalantar-Zadeh K. Patient education for phosphorus management in chronic kidney disease. Patient Prefer Adherence. 2013;7:379-90.

19. Kalantar-Zadeh K, Gutekunst L, Mehrotra R, et al. Understanding sources of dietary phosphorus in the treatment of patients with chronic kidney disease. Clin J Am Soc Nephrol. 2010;5(3):519-30.

20. Ramirez JA, Emmett M, White MG, et al. The absorption of dietary phosphorus and calcium in hemodialysis-patients. Kidney Int. 1986;30:753-9.

21. Weinman EJ, Light PD, Suki WN. Gastrointestinal phosphate handling in CKD and its association with cardiovascular disease. Am J Kidney Dis. 2013;62:1006-11.

22. Tonelli M. Serum phosphorus in people with chronic kidney disease: you are what you eat. Kidney Int. 2013;84(5):871-3.

23. Léon JB, Sullivan CM, Sehgal AR. The prevalence of phosphoruscontaining food additives in top-selling foods in grocery stores. J Ren Nutr. 2013;23(4):265-70.

24. Sherman RA, Ravella S, Kapoian T. A dearth of data: the problem of phosphorus in prescription medications. Kidney Int. 2015;87(6):1097-9.

25. Cupisti A, D’Alessandro C. Impatto dei componenti noti e non noti della dieta sull'apporto di fosforo. G Ital Nefrol. 2011;28(3):278-88.

26. Moe SH, Zidehsarai MP, Chambers MA, et al. Vegetarian compared with meat dietary protein source and phosphorus homeostasis in chronic kidney disease. Clin J Am Soc Nephrol. 2011;6:257-64.

27. Taylor LM, Kalantar-Zadeh K, Markewich T, et al. Dietary egg whites for phosphorus control in maintenance haemodialysis patients: a pilot study. J Ren Care. 2011;37:16-24.

28. Cupisti A, Gallieni M, Rizzo MA, Caria S, Meola M, Bolasco P. Phosphate control in dialysis. Int J Nephrol Renovasc Dis.
2013;6:193-205

29. Vrdoljak I, Krbavčic IP, Bituh M, Vrdoljak T, Dujmic Z. Analysis of different thermal processing methods of foodstuff to optimize protein, calcium, and phosphorus content for dialysis patient. J Ren Nutr. 2015;25(3):308-15.

30. Ogawa T, Shimada M, Nagano N, et al. Oral administration of Bifidobacterium longum in a gastro-resistant seamless capsule decreases serum phosphate levels in patients receiving haemodialysis. Clin Kidney J. 2012;5:373-4.

31. Caldeira D, Amaral T, David C, et al. Educational strategies to reduce serum phosphorus in hyperphosphatemic patients with chronic kidney disease: systematic review with meta-analysis. J Ren Nutr. 2011;21:285-94.

32. Pierratos A. Daily hemodialysis. Curr Opin Nephrol Hypertens. 2000;9(6):637-42.

33. Achinger SG, Ayus JC. The role of daily dialysis in the control of hyperphosphatemia. Kidney Int Suppl. 2005;95:S28-S32.

34. Spalding EM, Chamney PW, Farrington K. Phosphate kinetics during hemodialysis: Evidence for biphasic regulation. Kidney Int. 2002;61(2):655-67.

35. Minutolo R, Bellizzi V, Cioffi M, et al. Postdialytic rebound of serum phosphorus: pathogenetic and clinical insights. J Am Soc Nephrol. 2002;13:1046-54.

36. Lornoy W, De Meester J, Becaus I, Billiouw JM, Van Malderen PA Van Pottelberge M. Impact of convective flow on phosphorus removal in maintenance hemodialysis patients. J Ren Nutr. 2006;16(1):47-53.

37. Block GA, Raggi P, Bellasi A, Kooienga L, Spiegel DM. Mortality effect of coronary calcification and phosphate binder choice in incident hemodialysis patients. Kidney Int. 2007;71(5):438-41.

38. Kovesdy CP, Kuchmak O, Lu JL, Kalantar-Zadeh K. Outcomes associated with phosphorus binders in men with non-dialysisdependent CKD. Am J Kidney Dis. 2010;56(5):842-51.

39. Cannata-Andía JB, Fernández-Martin JL, Locatelli F, et al. Use of phosphate-binding agents is associated with a lower risk of mortality. Kidney Int. 2013;84(5):998-1008.

40. Palmer SC, Gardner S, Tonelli M, et al. Phosphate-binding agents in adults with CKD: a network meta-analysis of randomized trials. Am J Kidney Dis. 2016 Jul 22. pii: S0272-6386(16)30253-0.

41. Jamal SA, Vandermeer B, Raggi P, et al. Effect of calcium-based versus non-calcium-based phosphate binders on mortality in patients with chronic kidney disease: an updated systematic review and meta-analysis. Lancet. 2013;382(9900):1268-77.

42. Genovesi S, Gallieni M. Cardiovascular complications of calcium supplementation in chronic kidney disease: are there arrhythmic risks? Expert Opin Drug Saf. 2014;13(9):1143-8.

43. Malberti F. Hyperphosphataemia: treatment options. Drugs. 2013;73:673-88.

44. Blacher A, Guerin AP, Pannier B, Marchais SJ, London GM. Arterial calcifications, arterial stiffness, and cardiovascular risk in end-stage renal disease. Hypertension. 2001;38(4);938-42.

45. Chiu YW, Teitelbaum I, Misra M, et al. Pill burden, adherence, hyperphosphatemia, and quality of life in maintenance dialysis patients. Clin J Am Soc Nephrol. 2009;4(6):1089-96.

46. Karamanidou C, Clatworthy J, Weinman J, Horne R. A systematic review of the prevalence and determinants of nonadherence to phosphate binding medication in patients with end-stage renal disease. BMC Nephrol. 2008;9:2.

47. Sheikh MS, Maguire JA, Emmett M, et al. Reduction of dietary phosphorus absorption by phosphorus binders. A theoretical, in vitro, and in vivo study. J Clin Invest. 1989;83:66-73.

48. Block GA, Wheeler DC, Persky MS, et al. Effects of phosphate binders in moderate CKD. J Am Soc Nephrol. 2012;23:1407-15.

49. Fernández-Martin JL, Carrero JJ, Benedik M, et al. COSMOS the dialysis scenario of CKD-MBD in Europe. Nephrol Dial Transplant. 2013;28(7):1922-35.

50. Sherman RA. Hyperphosphatemia in dialysis patients: beyond nonadherence to diet and binders. Am J Kidney Dis. 2016;67(2):182-6 